\title{
Propuesta de indicadores para el estudio de los derechos de la infancia desde la Convención de los Derechos del Niño
}

\author{
Neubauer Esteban, Adrián \\ Grupo de Investigación sobre Políticas Educativas Supranacionales, Madrid, España \\ Adrian.neubauer@outlook.com
}

\section{Resumen}

Los derechos de la infancia tienen escaso estudio y repercusión en la Educación Comparada (Dávila Balsera, P. y Garmendia Naya, L. M., 2006), por diversas razones como la invisibilidad de los sectores vulnerables y la dificultad de acceso a informaciones de tipo cualitativo y cuantitativo en ciertas ocasiones en la materia.

De tal modo, en este trabajo, pretendemos aportar un conjunto de parámetros e indicadores que nos permitan llevar a cabo un estudio comparado a nivel supranacional, estatal o autonómico. Estos indicadores están directamente vinculados con la Convención de los Derechos del Niño, tratado e hito que podría calificarse como uno de los más importantes de la historia, tanto por su contenido, como por su gran apoyo a nivel mundial. Asimismo, este instrumento de evaluación se ha diseñado utilizando cada artículo como un parámetro, desglosado en diferentes indicadores, que a su vez, reflejan la vinculación con los diferentes artículos, no pudiendo olvidar el carácter holístico e integral de la Convención de los Derechos del Niño. Además, este instrumento cuenta con una escala Likert para el análisis de los datos y poder dar mayor profundidad al análisis. En definitiva, esta propuesta metodológica persigue la necesidad y el deseo de visibilizar a aquellos sectores más vulnerables en la sociedad, que actualmente son silenciados e invisibilizados por las estadísticas, impidiéndoles luchar y reclamar sus derechos, y la Educación Comparada, es un gran arma para la emancipación de los oprimidos.

\section{Abstract}

Infancy rights have a little and repercussion in Comparative Education (Dávila Balsera, P. y Garmendia Naya, L. M., 2006), for different reason as the invisibility of vulnerable sectors and the complexity of accessing to qualitative and quantitative information sometimes about the topic.

That way, in this researching, we pretend to contribute a bunch of parameters and indicators which allow as taking studies at supranational, national and autonomic level. There indicators are directly link to the Convention of the Rights of the Childs, treatment and milestone that could be calcified as one of the most important in the history, for its content, and for its world support. Likewise, this instrument of evaluation has been designed using every article as a parameter, slice in different indicators, that at their time, they reflect the link with the different articles, without forgetting the holistic and integral character of the Convention of the Rights of the Childs. Further, this instrument has a Likert scale for the analyse of the data and could realise a deeper analyses.

Definitely, this methodological proposal tries to attend to the necessity and the wish of make visible to more vulnerable sectors of the society, which actually they are muted and invisibilized for statistics, preventing them of fighting and claiming for their rights, and the Comparative Education, is a great weapon to emancipate to oppressed people.

Palabras clave: Derechos Humanos, Convención de los Derechos del Niño, Derechos de la Infancia, Metodología, Evaluación.

Keywords: Human rights, Convention of the Rights of the Childs, Infancy Rights, Methodology, Evaluation.

\section{INTRODUCCIÓN}

Los derechos de la infancia han evolucionado notable y positivamente a lo largo de los siglos XIX y XX. Sin embargo, su situación actual sigue siendo crítica, como consecuencia de los constantes conflictos bélicos que existen en el plano internacional. Este fenómeno, sumado a las características 
propias de los menores, les hacen ser un sector con gran riesgo de vulnerabilidad (Ravetllat Ballesté, I., 2015; Civarolo Arpón, M. M. y Fuentes Torresi, M. A., 2018).

Este hecho se agrava por la invisibilidad que padecen los menores de edad no acompañados y los menores en territorios nacionales ante la falta de estadísticas o la manipulación de las mismas. El acceso a información sobre menores de edad no acompañados es complejo, especialmente en el caso de las niñas, pues suelen estar a merced de las mafias y las redes de tráfico de persona.

En vista de la dramática situación que padecen los menores a lo largo del planeta, es preciso que la investigación se comprometa y alinee a favor de los derechos de la infancia. Una forma muy adecuada para hacerlo sería a través de la Educación Comparada. A pesar de ello, los derechos de la infancia no han irrumpido con gran fuerza en esta metodología investigadora (Dávila Balsera, P. y Garmendia Naya, L. M., 2006). Por este motivo, en este trabajo, presentaremos un instrumento de evaluación con una batería de indicadores que permita a los investigadores visibilizar la situación de los menores, con el fin de poder tener un impacto positivo en la creación de políticas sociales, migratorias y educativas.

\section{LOS DERECHOS DE LA INFANCIA DESDE LA EDUCACIÓN COMPARADA Y LA CONVENCIÓN DE 1989}

En septiembre de 1990 entró en vigor la Convención sobre los Derechos del Niño. Este nuevo tratado supranacional es el tratado más importante para proteger y promover los derechos de la infancia, tanto a nivel político, social, cultural, económico y civil (Dávila Balsera, P. y Garmendia Naya, L. M., 2010; Gillet-Swan, J. y Vicki Coppokc, 2016). De hecho, la Convención, ha sido el tratado con más apoyos a lo largo de la historia (Dávila Balsera, P. y Garmendia Naya, L. M., 2008; Dávila Balsera, P. y Garmendia Naya, L. M., 2010; Gillet-Swan, J. y Vicki Coppokc, 2016).

La Convención, supuso la culminación (hasta la fecha) de las declaraciones previas sobre los derechos de la infancia, la de Ginebra (1924) y los Derechos del Niño (1959). En el caso de las dos primeras declaraciones se enmarcaron y desarrollaron en contextos de conflictos armados y políticos, como la Primera Guerra Mundial, y a la guerra fría, respectivamente (Dávila Balsera, P. y Garmendia Naya, L. M., 2006). Sin embargo, la Convención, en palabras de Paulí Dávila y Luis María Naya (2006), debe ser enmarcada en un «contexto de fortalecimiento de los derechos humanos».

Es interesante pararse a reflexionar sobre la idea de que en un primer momento podríamos pensar que la Convención fue un tratado pensado y ratificado para atender a los derechos de la infancia en los países en vías de desarrollo (Dávila Balsera, P. y Garmendia Naya, L. M., 2009). Asimismo, no podemos olvidar que los derechos de la infancia son vulnerados en todos los países del mundo de un modo u otro, he ahí la importancia de la autoridad, la universalidad y las obligaciones estatales que promueve la Convención (John I’Anson en Gillet-Swan, J. y Vicki Coppokc, 2016).

La Convención tiene una línea continuista respecto a sus predecesoras en el marco de los derechos de la infancia. De tal modo, sus principales valores se podrían resumir en: 1) el derecho a la vida y al pleno desarrollo de la persona; 2) garantizar los derechos y libertades civiles y políticas; 3) el principio de no-discriminación; 4) y en último lugar, la gran aportación de dicho tratado, el interés superior del menor (Dávila Balsera, P. y Garmendia Naya, L. M., 2006; Dávila Balsera, P. y Garmendia Naya, L. M., 2008; Dávila Balsera, P. y Garmendia Naya, L. M., 2009; Dávila Balsera, P. y Garmendia Naya, L. M., 2010; Gwenneth Phillips L., en Gillet-Swan, J. y Vicki Coppokc, 2016).

Sin embargo, todavía el discurso de los derechos de la infancia en Educación Comparada es muy escaso o nulo (Dávila Balsera, P. y Garmendia Naya, L. M., 2006). Asimismo, entendiendo a los menores como sujetos de derechos, es preciso abrir nuevas líneas de investigación en esta metodología con el fin de fortalecerla (Dávila Balsera, P. y Garmendia Naya, L. M., 2006). Por otro lado, también es preciso arrojar luz sobre la realidad de los menores de edad en los sistemas educativos, dando la importancia que merece al derecho a la educación, pues es un derecho vertebrador para los demás (Dávila Balsera, P. y Garmendia Naya, L. M., 2006).

Por estas razones y con motivo de visibilizar y promover los derechos de la infancia desde la Educación Comparada, hemos diseñado este instrumento de evaluación a partir de la Convención. 


\section{INSTRUMENTO DE EVALUACIÓN}

\subsection{Contenido y presentación del instrumento de evaluación}

Este instrumento de evaluación fue construido a partir de la lectura y la revisión analítica de la Convención de los Derechos del Niño (1989), de modo que a partir de los artículos y principios recogidos en dicho documento, se pudieran extrapolar las dimensiones, los parámetros e indicadores. La valoración de este instrumento es de carácter cualitativo y se desarrollará a través de una escala Likert (1=totalmente en desacuerdo; 2=en desacuerdo; 3=ni acuerdo en desacuerdo; 4=de acuerdo; 5=totalmente de acuerdo).

Para ello, se llevaron a cabo diversas categorías fundamentadas en los principios y artículos de la Convención, haciendo un total de seis dimensiones, las cuales de forma más detallada presentaremos y desglosaremos en sus parámetros e indicadores correspondientes, a la vez que los vinculamos con su artículo de referencia en la Convención:

1. No-discriminación (Art. 2) (Dimensión):

1.1. Discriminación (Parámetro)

1.1.1. Existe el principio de educación inclusiva en la legislación educativa (2) (Indicador)

1.1.2. La ley recoge la multiculturalidad como un elemento positivo y enriquecedor (2)

1.2. Lenguaje

1.2.1. Existen leyes contra el comportamiento (lenguaje, actitud, actividades) sexistas en la escuela (2)

1.2.2. Existen leyes contra el comportamiento (lenguaje, actitud, actividades) racista o los prejuicios en la escuela (2)

1.3. Medidas preventivas

1.3.1. Se promueve la realización de actividades a favor de la multiculturalidad desde la ley educativa (2)

1.3.2. Desde la legislación educativa se promueven los grupos de trabajo interactivos y flexibles (2)

2. Derechos y obligaciones para la protección contra los malos tratos (Arts. 3 y 19)

2.1. Seguridad

2.1.1. El aula cumple las medidas de seguridad según la normativa vigente (3)

2.1.2. Se prohíben los castigos corporales (3 y 19)

2.1.3. Se condena el trato vejatorio y ofensivo por parte del docente sobre el alumnado (3 y 19)

2.1.4. Porcentaje de bullying en la escuela (3 y 19)

2.1.5. Los centros educativos tienen medidas para la resolución de situaciones violentas (3 y 19)

2.1.6. El Estado tiene medidas de prevención explícitas contra el bullying (3 y 19)

3. Libertad de expresión (Arts. 12, 13 y 17)

3.1. Derecho a expresar su opinión

3.1.1. Existe un medio o institución para que el alumnado exprese su opinión libremente (12)

3.1.2. Existen espacios y momentos para que el alumnado exprese su opinión de forma sistemática (12)

3.1.3. La participación activa del alumnado se recoge en la legislación educativa como algo positivo y obligatorio (12) 
3.2. Reconocimiento de su opinión

3.2.1. El alumnado tiene capacidad y potestad para tomar decisiones relacionadas con la práctica docente

3.2.2. El alumnado tiene capacidad y potestad para tomar decisiones relacionadas con la normativa del aula

\subsubsection{1. (12)}

3.2.3. El alumnado tiene capacidad y potestad para tomar decisiones relacionadas con la organización y normativa del centro (12)

3.3. Buscar, recibir y difundir información y la posibilidad de acceder a los medios de comunicación críticamente

3.3.1. El alumnado cuenta con mecanismos e instrumentos para expresar su opinión de forma sistemática (13)

3.3.2. Se promueve la búsqueda autónoma de información crítica siguiendo el criterio de validez y reflexión crítica, por parte del alumnado (13 y 17)

3.3.3. La legislación educativa invita al docente a promover el análisis crítico de los medios de comunicación y la información que transmiten (13 y 17)

4. Libertad (Arts. 14 y 15)

4.1. Libertad de pensamiento

4.1.1. Se valora y reconoce la diversidad de ideas en la comunidad educativa (14)

4.1.2. El alumno puede espresar su opinión libremente sin efectos negativos(14)

4.2. Libertad religiosa

4.2.1. El alumnado puede practicar su fe libremente en el centro (14)

4.2.2. El alumnado puede estudiar su religión en el sistema educativo formal (14)

4.2.3. El alumnado conoce y valora la diversidad religiosa existente (14)

4.3. Libertad de asociación

4.3.1. El alumnado dispone de espacios y tiempos dentro del horario lectivo para reunirse (15)

4.3.2. El Estado y los centros educativos informan al alumnado sobre el derecho de libre asociación de los mismos (15)

4.3.3. Porcentaje y peso de las decisiones del alumnado en el centro escolar (15)

4.3.4. Existen asociaciones de estudiantes formadas por ellos mismos (15)

5. Menores de edad refugiados (Art. 22)

5.1. Atención especial

5.1.1. La legislación trata de forma explícita como contenido la situación de los niños refugiados (22)

5.1.2. La legislación trata de forma explícita como contenido la situación de los menores no acompañados (22)

6. Educación $(23,29$ y 31)

6.1. Medidas de atención a personas con discapacidad

6.1.1. La ley educativa recoge los principios de normalidad e inclusión como pilares (23)

6.1.2. El docente debe llevar a cabo medidas didácticas inclusivas y de atención a la diversidad (23)

6.1.3. Existen centros educativos especiales para personas con discapacidad (23)

6.2. Desarrollo de la identidad cultural propia

6.2.1. El docente debe promover actividades para conocer y valorar la cultura propia (29)

6.2.2. Se favorecen actividades culturales en el entorno urbano desde la legislación educativa (29) 
6.3. Derecho al juego

6.3.1. Las actividades de aprendizaje tienen un carácter lúdico (31)

6.3.2. El juego es la principal metodología que ha de utilizar el docente según la legislación educativa (31)

6.3.3. Se promueven actividades diversas y atractivas para el alumnado (31)

6.4. Actividades artísticas y expresivas

6.4.1. El alumnado puede expresar sus ideas y sentimientos a través del cuerpo, el movimiento y el lenguaje oral (31)

6.4.2. El alumnado desarrolla las capacidades artístico-expresivas de su cuerpo (31)

6.4.3. Se llevan a cabo actividades musicales en el centro educativo (31)

6.4.4. Se utiliza la pintura como medio de expresión de sentimientos por parte del alumnado (31)

\subsection{Objetivos}

Los objetivos que persigue este instrumento de evaluación son los siguientes:

1. Visibilizar los derechos de la infancia en las políticas educativas.

2. Identificar elementos que coarten o vayan en contra de los derechos de la infancia en las políticas educativas.

3. Permitir la comparación entre los diferentes Estados en materia de los derechos de la infancia en sus políticas y sus sistemas educativos.

\subsection{Instrucciones de uso}

Este instrumento debe de ser cumplimentado por un experto en derechos humanos y en educación, o bien por dos expertos, uno de cada campo para que complementen su visión y análisis. En último lugar, se deberán tomar como referencia los documentos educativos y organizativos del centro, además de las legislaciones estatales, autonómicas y/o provinciales correspondientes.

\section{DISCUSIÓN Y CONCLUSIONES}

A lo largo de este trabajo se han presentado una serie de parámetros e indicadores con el fin de dar mayor notoriedad y visibilidad a los derechos de la infancia. La elaboración de instrumentos así, siguiendo con la obra de Luis María Naya y Paulí Dávila (2006) se hace cada día más urgente debido al auge de los organismos internacionales como agentes clave para impulsar políticas supranacionales, internacionales y estatales para promover y garantizar los derechos de los niños y las niñas del mundo.

Para ello, la Educación Comparada debe alinearse a favor de esta problemática, pues puede ser de gran utilidad para denunciar las precarias condiciones de vida de muchos menores, o cómo éstos son incapaces de ejercer sus derechos ante las barreras que los Estados y la sociedad les imponen. En definitiva, en una sociedad globalizada donde la información es constante y abundante, la Educación Comparada debe luchar para arrojar luz sobre los derechos de los menores en las estadísticas, que en muchas ocasiones silencian sus voces y derechos. 


\section{REFERENCIAS BIBLIOGRÁFICAS}

Dávila Balsera, P. y Garmendia Naya, L. M. (2008). El discurso proteccionista sobre los derechos de la infancia en los Tratados Internacionales. XXI. Revista de Educación, Universidad de Huelva, 10.

Dávila Balsera, P. y Garmendia Naya, L. M. (2010). Infancia, Educación y Códigos de la Niñez en América Latina. Un análisis comparado. Revista Española de Educación Comparada, (16), 213-233.

Organización de las Naciones Unidas (1989). Convención sobre los Derechos del Niño. Obtenido el 12 de abril de 2018 desde http://www.un.org/es/events/childrenday/pdf/derechos.pdf.

Ravetllat Ballesté, I. (2015). Aproximación histórica a la construcción sociojurídica de la categoría infancia. Editorial Universitat Politècnica de València.

Civarolo Arpón, M. M. y Fuentes Torresi, M. A (2018). Miradas de educadores y padres sobre la infancia actual. Revista Latinoamericana de Educación Infantil, 2(3), 145-160.

Dávila Balsera, P. y Garmendia Naya, L. M. (2006). Los derechos de la infancia en el marco de la educación comparada. Revista de educación, 340, 1009-1038.

Dávila Balsera, P. y Garmendia Naya, L. M. (2009). El derecho a la educación en Europa: una lectura desde los derechos del niño. Bordón. Revista de pedagogía, 61(1), 61-76.

Gillett-Gwan, J. \& Coppock, V., (2016). Children's rights, educational research and the UNRCR. Past, present and future. Symposium books; $166 \mathrm{pp}$. 